\title{
ALLA RICERCA \\ DELLA COLLEGIALITÀ DI GOVERNO: \\ I VERTICI DI MAGGIORANZA DAL 1970 AL 1994
}

\author{
di Annarita Criscitiello
}

\section{Premessa}

Le radici della debolezza del governo collegiale nell'Italia repubblicana sono state individuate nel modello costituzionale formale così come fu ideato dai padri fondatori ${ }^{1}$. Ma anche nel fatto che i governi italiani, tranne qualche eccezione, sono sempre stati governi di coalizione, una condizione che necessariamente rende il processo decisionale collegiale molto più complicato e controverso (Pappalardo 1978; Bogdanor 1983; Pridham 1986; Budge e Keman 1990; Laver e Schofield 1990).

In particolare, il fatto che il governo italiano è un governo di coalizione ha sempre avuto un effetto immediato sulla formazione dell'agenda dell'esecutivo. I temi discussi in sede di Consiglio dei Ministri sono, in generale, i temi posti dai partiti della coalizione piuttosto che da una amministrazione dominata dall'ufficio del premier, come in Germania (Müller-Rommel 1988) o come in Francia, dove «le riunioni del Consiglio dei Ministri sono precedute da incontri più ristretti e informali che riuniscono ministri ed alti funzionari e nelle quali si determina l'essenziale delle decisioni» (Mény 1990, 400).

L'eterogeneità partitica delle coalizioni di governo mal si presta però ad essere analizzata attraverso le rigide lenti della dottrina costituzionale. Così gran parte del dibattito - prevalentemente giuridico - si è concentrato sul problema della relazione del premier col gabinetto, così come peraltro è accaduto in

Desidero ringraziare il prof. Mauro Calise per $i$ suoi preziosi e stimolanti suggerimenti che non sono mai mancati in tutte le fasi della ricerca.

1 Tra gli autori che più recentemente hanno evidenziato le lacune della Costituzione manifestando l'esigenza di modificarla, anche se da diversa prospettiva, cfr. Sartori (1994), Rebuffa (1995) e Cassese (1995).

RIVISTA ITALIANA DI SCIENZA POLITICA / a. XXVI, n. 2, agosto 1996 
Gran Bretagna dove «la polarizzazione tra due campi, uno che asseriva la capacità di gestione collegiale vs i ministri di gabinetto e l'altro che enfatizzava la predominanza del primo ministro, ha artificiosamente limitato il campo di studio» (Dunleavy e Rhodes 1990, 5).

Sono così rimasti fuori da una sistematica osservazione empirica proprio quei meccanismi compensativi che il sistema istituzionale aveva finito col mettere in opera per risolvere alcune contraddizioni tra un debole impianto costituzionale formale e le pretese dei partiti che compongono le coalizioni di governo. In Italia, la collegialità dell'indirizzo politico nell'attività di governo si è infatti presentata - quando c'è stata - come prodotto di sedi extra-costituzionali piuttosto che degli organismi a ciò formalmente deputati, quali il Consiglio dei Ministri o la sua Presidenza.

Tali sedi extra-costituzionali - $\mathrm{i}$ «Vertici di maggioranza» sono ben note alla cronaca politica e sono state oggetto, in più occasioni, di critiche legate appunto al ruolo di supplenza che hanno assunto nei confronti del governo ufficiale. Si tratta di uno strumento informale di governo che è diventato prassi col passare del tempo. Nonostante però i Vertici di maggioranza siano stati spesso menzionati dalla letteratura giuridica tra gli organi della costituzione materiale (Ferrara 1973; Lavagna 1974; Capotosti 1975; Cuccodoro 1983; Calandra 1986) e compaiano con regolarità nelle cronache politiche riportate dalla rivista ufficiale della Presidenza dei Ministri ${ }^{2}$, non ne sono mai state definite più puntualmente la fisionomia e le procedure.

In questo articolo analizzerò - nella prima parte - i Vertici di maggioranza che si sono succeduti in Italia nel ventennio 1970-1992, mettendone in luce le principali caratteristiche e i mutamenti intercorsi ${ }^{3}$. Passerò poi brevemente in rassegna i governi della fase cosiddetta di «transizione istituzionale», durante

2 Il mensile «Vita Italiana», pubblicato a cura del Dipartimento per l'Editoria presso la Presidenza del Consiglio dei Ministri, riporta nel calendario politico le date e le informazioni più salienti dei Vertici di maggioranza. Non di rado, inoltre, nelle pagine di cronaca politica si possono trovare dettagliati articoli sui contenuti e l'esito degli incontri.

3 Pur avendo tenuto conto delle informazioni ufficiali fornite da «Vita Italiana», ho preferito basarmi su una fonte neutrale. I dati qui presentati risultano dallo spoglio delle pagine quotidiane del «Corriere della Sera» per il periodo 1970-1994. Alcuni primi risultati, limitatamente al periodo $1970-1989$, sono stati pubblicati in Criscitiello (1993). 
la quale i Vertici sembravano destinati ad estinguersi e, infine, la nascita della cosiddetta Seconda Repubblica che invece - come vedremo - almeno sotto questo profilo, non si preannuncia molto diversa dalla Prima. Dopo un breve intermezzo «tecnico», con il governo presieduto da Silvio Berlusconi, infatti, i Vertici faranno prepotentemente ritorno sulla scena politica italiana.

\section{Una proposta di classificazione}

Il Vertice di maggioranza riunisce, oltre al presidente del Consiglio, i segretari dei partiti che formano la maggioranza di governo. Mentre questi ultimi sono sempre presenti, è possibile che saltuariamente vi prendano parte anche $i$ capigruppo parlamentari, i vicesegretari o $\mathrm{i}$ «tecnici» dei partiti e, a volte, anche alcuni ministri: solitamente quelli posti a capo di dicasteri «strategici» come quelli economici.

Una prima distinzione tra i Vertici può essere ricondotta alla situazione politica generale nella quale essi si svolgono e ai temi politici trattati.

Per quanto concerne lo scenario politico generale che fa da sfondo a questi incontri, a seconda che un governo sia in carica oppure in fase di formazione in seguito ad una crisi, si può distinguere tra «Vertici ordinari» e «Vertici di crisi». Il primo tipo di Vertice, quello «ordinario», si svolge quando l'esecutivo è nel pieno delle sue funzioni e costituisce una delle sedi deputate alla contrattazione tra i partiti che formano la maggioranza di governo, che qui verificano l'esistenza o la validità dell'accordo di coalizione nonché lo stato di attuazione del programma di governo.

Il secondo tipo di Vertice, che abbiamo definito «di crisi», si svolge invece quando è in fase di formazione un nuovo governo. Esso è un meccanismo pressoché indispensabile ai partiti della futura coalizione tanto per la risoluzione definitiva della crisi che ha determinato la caduta del vecchio esecutivo, quanto per la nascita del nuovo. Inoltre, se da un lato i Vertici di crisi condividono con quelli ordinari il fatto di costituire la principale sede di contrattazione sui contenuti delle coalizioni, dall'altro essi si caratterizzano per essere non solo il luogo privilegiato per la definizione del programma politico del nuovo governo, ma anche il luogo in cui vengono ripartiti i nuovi incarichi ministeriali. 
Proprio a proposito dei temi che sono in discussione durante questi incontri, ci è sembrato opportuno dividere i Vertici ordinari in «monotematici» o «pluritematici» a seconda che sul tavolo delle trattative fossero poste una o più questioni controverse.

I Vertici ordinari pluritematici sono, nella maggior parte dei casi, la sede del confronto politico all'interno della coalizione di governo. Tale tipo di Vertice, conosciuto anche come «verifica della maggioranza», è di fatto diventato un'arena particolare di contrattazione e negoziazione tra i segretari dei partiti che può influenzare la durata stessa di un governo ${ }^{4}$. Una chiarificazione tra i partiti della maggioranza è infatti il primo gradino con il quale si cerca di ricomporre i contrasti quando ad uno o più partner sembrano venire meno le condizioni poste alla base dell'accordo di coalizione e, quindi, del buon funzionamento dell'esecutivo. Come ha scritto uno dei protagonisti della vita politica italiana, «vi sono parole magiche nel vocabolario esistenziale dei governi, con qualche variante ma con significato univoco. Quando si comincia a parlare della necessità di tonificazioni, rilanci, verifiche e simili il barometro segna minacciosamente al peggio» (Andreotti 1991, 198).

E vero infatti che, molto spesso, questo tipo di Vertice cerca di evitare che una crisi politica divenga una crisi istituzionale e cioè, in pratica, un Vertice ordinario è spesso convocato al fine di prevenire una crisi di governo. Naturalmente il tentativo può risultare infruttuoso, col risultato che in sede di verifica della maggioranza vengano addirittura decise le dimissioni del governo. È stato questo il caso, ad esempio, del Vertice del 15 gennaio 1972, allorché i quattro partiti che formavano la maggioranza (democristiani, socialisti, socialdemocratici e repubblicani) si riunirono dopo reiterate richieste di una verifica della coalizione del centro-sinistra da parte del PSDI. In quella occasione si discusse collegialmente la decisione dei repubblicani di ritirare il loro appoggio al governo e si finì col concordare le dimissioni dell'allora presidente del Consiglio, Emilio Colombo.

Se, dunque, in governi di coalizione - come sono la maggio-

4 Naturalmente, l'espressione «verifica di maggioranza», con la quale viene solitamente indicata dai media la maggior parte dei Vertici che noi abbiamo definito ordinari pluritematici, deve essere distinta dall'omonimo meccanismo formalmente previsto dai regolamenti parlamentari: in questo caso, infatti, è proprio il governo a richiedere la verifica della maggioranza per accertare se esso gode ancora della fiducia del Parlamento. 
ranza di quelli che si sono succeduti per oltre un quarantennio in Italia - è intuitivo pensare a sedi di contrattazione partitica extragovernative nelle quali vengono verificati precedenti accordi di governo o se ne formano di nuovi, meno scontato è scoprire, però, come tali Vertici siano diventati la sede decisionale vera e propria di innumerevoli provvedimenti, il «vero dominus della compagine governativa» (Rolla 1982, 387), sostituendo, di fatto, l'organo costituzionalmente deputato a governare. Infatti, come vedremo, non tutti i Vertici pluritematici sono delle semplici verifiche di maggioranza: in sede di Vertice ordinario vengono anche discusse scelte relative a policies specifiche, poi soltanto ratificate dal Consiglio dei Ministri.

La discussione su una policy particolare ricorre sempre, per definizione, nel Vertice ordinario monotematico che si svolge appunto quando una issue di cruciale importanza richiede, da sola, la verifica o la contrattazione delle posizioni di tutti i partiti partecipanti al governo.

Accanto alla distinzione tra Vertici di crisi e Vertici ordinari e, all'interno di questi, tra Vertici monotematici e pluritematici, ciascun Vertice è stato poi analizzato sulla base delle seguenti variabili: il proponente, i partecipanti, la sede, la durata e l'esito.

La prima variabile, il proponente, si riferisce al partito che per primo ritiene necessario un confronto; il partito, cioè, almeno pubblicamente più interessato a verificare la condizione dell'accordo di coalizione o a discutere su una o più issues. Si tratta di una variabile che riguarda in questi termini soltanto $i$ Vertici ordinari giacché per quelli di crisi si dovrà parlare di vera $\mathrm{e}$ propria «convocazione» dei partecipanti da parte del presidente incaricato di formare il nuovo governo, più che di semplice proposta.

La variabile che identifica chi prende parte a questo tipo di incontri, quella cioè dei partecipanti al Vertice, ci permette invece di distinguere tra attori solo partitici - quelli cioè che in questa sede rivestono solo incarichi di partito - e attori anche governativi, ossia che prendono parte al Vertice in quanto componenti dell'esecutivo. Tra i primi vi sono sempre i segretari dei partiti che compongono la maggioranza, mentre gli altri attori partitici possono mutare sia nel numero che nel ruolo. Si tratta infatti a volte di vicesegretari, di coordinatori delle segreterie oppure degli esperti dei partiti in determinati settori. Spesso, soprattutto nel primo dei due decenni analizzati, prendevano parte ai Vertici anche i capigruppo alla Camera e al Senato dei 
partiti della maggioranza. Per quanto concerne invece i partecipanti che abbiamo classificato come «anche governativi», vi è innanzitutto il presidente del Consiglio che, tranne qualche rarissima eccezione, è sempre presente. Vi è poi il vicepresidente del Consiglio, qualche ministro (soprattutto quelli a capo dei dicasteri economici) e, raramente, qualche sottosegretario.

Un'altra variabile considerata è quella della durata del Vertice. Ciò, infatti, può essere un indicatore della complessità e quindi della difficoltà nella risoluzione delle questioni poste in campo dai partiti. La durata, naturalmente, non dovrà essere considerata in maniera rigida, guardando, cioè, meramente al numero delle ore o dei giorni degli incontri, giacché i Vertici, spesso, non sono che l'approdo di un flusso di negoziazioni più informali, incontri bilaterali, contatti telefonici, ecc. lungo un certo periodo di tempo. Ciascun Vertice, dunque, va considerato come il risultato di un processo di contrattazione partiticogovernativa che va ben al di là delle ore o dei giorni ad esso specificamente dedicati.

L'ultima variabile, quella che riguarda l'esito dei Vertici, indica se questi incontri si concludono con un accordo della maggioranza o se invece falliscono. Considereremo dunque questa variabile come un indicatore del livello di coesione tra i partiti della coalizione e, indirettamente, della buona o cattiva salute dell'esecutivo.

\section{Vertici del ventennio 1970-1992}

I Vertici di maggioranza che risultano dalla nostra rilevazione sulle pagine del «Corriere della Sera» dal 1970 al 1992 sono 85, 66 Vertici ordinari e 19 Vertici di crisi. I 19 Vertici di crisi coprono un arco complessivo di 27 governi. Non tutte le crisi di governo, dunque, hanno avuto un Vertice di crisi: qualche volta, infatti, è accaduto che gli accordi di coalizione per il nuovo esecutivo si siano raggiunti nei ben noti «incontri bilaterali» tra il presidente del Consiglio incaricato e i segretari dei partiti intenzionati a far parte della maggioranza di governo. La gran parte dei governi dell'Italia repubblicana qui considerati - 19 esecutivi su 27 - pronti a ricevere la fiducia del Parlamento, sono stati invece il risultato di un processo di contrattazione prevalentemente gestito nei Vertici di crisi. Gestito cioè da un organismo collegiale extracostituzionale convocato e presieduto 


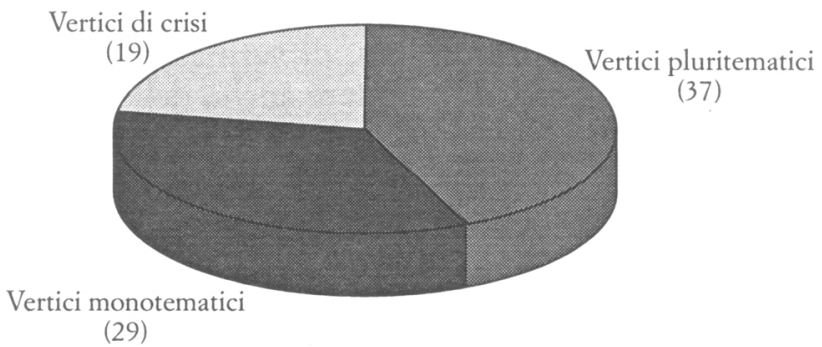

FIG. 1. Distribuzione dei Vertici di maggioranza per tipo.

sempre dal presidente del Consiglio incaricato, caratterizzato dunque da un espresso livello di formalizzazione.

Nel caso di un Vertice di crisi, come si è accennato, ci si accorda sul nuovo governo. Si discute quindi tanto della cosiddetta «formula» politica - ossia della composizione partitica, delle nuove alleanze o del ripristino delle precedenti - quanto del programma politico e, ovviamente, della distribuzione degli incarichi - dei ministri così come dei sottosegretari. Mentre in alcuni casi la discussione sulla formula politica del nuovo esecutivo prevale sugli aspetti sostanziali programmatici, in molti altri casi i partecipanti al Vertice di crisi pongono maggiore attenzione alle questioni da affrontare una volta in carica. E come se ci fossero due vere e proprie «metodologie» per risolvere le crisi di governo, a seconda che prevalga la formula sul contenuto o viceversa, costituendo ciò quella che è stata definita la «caratteristica pendolare delle crisi» (Andreotti 1991, 208).

L'agenda dei Vertici di maggioranza che abbiamo definito ordinari è invece molto più complessa. I Vertici ordinari, infatti, non servono semplicemente a verificare gli accordi di coalizione: essi molto spesso diventano la sede di discussione dei temi dell'agenda governativa limitando - di fatto - l'autonomia decisionale dell'esecutivo. Dei 66 Vertici ordinari rilevati, 29 sono stati monotematici - dedicati, cioè ad una sola issue mentre 37 hanno discusso di più problemi o hanno semplicemente verificato l'accordo di coalizione.

Le stesse verifiche, d'altro canto, vedono di frequente all'ordine del giorno tanto la riproposizione delle diverse formule di coalizione - in una sorta di rinnovato giuramento di reciproca collaborazione - quanto la concreta riformulazione delle diverse posizioni dei partiti su punti specifici del programma di gover- 
no. Nel secondo caso, dunque, i singoli problemi vengono riesaminati con l'intento, da un lato, di verificarne lo stato di attuazione, dall'altro, però, di prendere anche delle vere e proprie decisioni in merito. Non tutti i Vertici pluritematici, infine, sono delle verifiche politiche: in molti casi si tratta di incontri nei quali l'accordo sulla formula della coalizione e sul programma di governo è dato per scontato e quindi si ritiene opportuno discutere determinate issues prima di farlo in sede di Consiglio dei Ministri.

È successo così - ad esempio - in un Vertice dell'ottobre del 1970 quando, oltre ad alcune misure economiche, furono anche decisi specifici provvedimenti da adottare in merito alla «questione calabrese» che riguardava la scelta del capoluogo tra Reggio Calabria e Catanzaro; oppure in un Vertice del gennaio dell'anno seguente, durante il quale si discusse genericamente della situazione economica ma si presero anche importanti decisioni in merito alle presidenze degli enti pubblici. Nel Vertice del 16 maggio 1973 si passò dagli aumenti di stipendio dell'alta burocrazia, alla TV via cavo, al tentativo di scongiurare una serie di scioperi del pubblico impiego. Ancora, in un Vertice definito «segretissimo», svoltosi alla fine del 1980, nell'ufficio privato dell'allora presidente del Consiglio Forlani, in sole quattro ore si discusse del sequestro del giudice D'Urso ad opera delle Brigate Rosse, dei provvedimenti da prendere nel Mezzogiorno a seguito del terremoto, ma si decise anche la sostituzione del ministro Bisaglia e... che le nomine bancarie non sarebbero più state fatte in sede di Vertice. Quattro anni dopo saranno invece le nomine dei dirigenti RAI ad essere discusse in un Vertice pluritematico insieme ad un decreto governativo definito «antinflazione», alla campagna elettorale per le elezioni europee e alla composizione della giunta comunale di Napoli.

Nel caso dei Vertici monotematici, invece, sono singoli temi ad essere ritenuti importanti al punto tale da richiedere un apposito incontro. Circa la metà dei Vertici che abbiamo rilevato ha trattato temi di carattere economico: dalle questioni genericamente riportate dalla stampa come provvedimenti economico-finanziari a specifiche issues, quali progetti di legge sulla casa, l'ingresso dell'Italia nello SME, la riforma pensionistica, la scala mobile, i cosiddetti ticket sanitari. Quelli non economici sono stati invece dedicati a problemi di governo locale, in generale alla libertà di alleanze amministrative divergenti dalla formula di governo e in particolare, ad esempio, alla giunta regio- 
nale sarda o alla questione altoatesina; a problemi di politica internazionale come la definizione dei rapporti diplomatici con la Cina Popolare o il resoconto del presidente del Consiglio fatto dopo una importante visita negli Stati Uniti o, infine, a problemi squisitamente interni come la cosiddetta questione morale o l'ordine pubblico. Su questi ultimi due temi, ad esempio, si sono tenuti ben sette Vertici a cavallo tra gli anni '70 e '80: tanto i provvedimenti da prendere in seguito al rapimento Moro, quanto le conseguenze del caso P2, portarono ad una notevole impennata di questi incontri monotematici.

A proposito dei partiti che per primi richiedono il Vertice, il primo dato interessante riguarda il partito di maggioranza relativa, la Democrazia Cristiana, che, da solo, ha proposto circa il $50 \%$ degli incontri. Non trascurabile, tuttavia, è il dato che riguarda i socialisti, con 11 proposte e i repubblicani che seguono con 9 proposte. Spadolini, nonostante la sua dichiarata avversione per i Vertici di maggioranza, ne convocò ben due in soli due mesi di presidenza. Con poche eccezioni, si tratta di Vertici tenuti quando i rispettivi segretari, Craxi e Spadolini, erano anche presidenti del Consiglio, a conferma del fatto che il partito che detiene la leadership governativa tende a farsi promotore degli incontri tesi a consolidare tale posizione. Inoltre, nel periodo 1984-1986, durante i governi presieduti da Craxi, i Vertici di maggioranza sono stati proposti direttamente dalla persona del presidente del Consiglio. Sebbene, dunque, Spadolini e Craxi siano state personalità con differenti stili politici nella conduzione del governo, si può ben essere d'accordo sul fatto che «i loro periodi di incarico sono tuttavia entrambi illuminanti nel contesto del tentativo di riabilitazione del ruolo del primo ministro proprio degli anni '80» (Hine e Finocchi 1991, 87). Un altro dato interessante riguarda gli anni 1976-1979. Durante il periodo della cosiddetta Grande Coalizione caratterizzata dalla partecipazione del partito comunista alla maggioranza parlamentare, fu proprio il PCI di Berlinguer che propose ben due Vertici pluritematici, più precisamente due verifiche tra il monocolore Andreotti e i partiti che, astenendosi in Parlamento al momento di votarne la fiducia, ne avevano consentito la formazione. Dunque, sebbene tradizionalmente all'opposizione, i comunisti non solo accettarono questo strumento tipico della gestione governativa, ma ne fecero un uso diretto. Già pochi mesi dopo l'avvìo del cosiddetto «governo della non sfiducia», i comunisti sollecitarono un incontro collegiale formalizzando 
addirittura la richiesta con una lettera inviata al presidente del Consiglio e agli altri partiti «astenuti». La DC però rifiutò controproponendo una serie di incontri bilaterali tra Andreotti e i segretari dei partiti che avrebbe dovuto precedere il dibattito alla Camera. Solo l'intensificarsi degli attacchi terroristici delle Brigate Rosse portò, nel dicembre dello stesso anno, ad un incontro collegiale, proposto questa volta dal neo-eletto segretario del PSI Bettino Craxi. Il summit, un Vertice monotematico dedicato alla difesa dell'ordine pubblico, vide la partecipazione oltre che del presidente del Consiglio Andreotti, del ministro della giustizia e del capo dei servizi di sicurezza, anche dei segretari dei sei partiti che formavano la maggioranza (DC, PSI, PCI, PLI, PRI e PSDI).

I media naturalmente posero tutti l'accento sul fatto che per la prima volta la DC non aveva sollevato obiezioni ad una seduta con i comunisti. In particolare, il «Corriere della Sera» dedicò un articolo in prima pagina proprio al risvolto politico dell'incontro e l'organo di stampa ufficiale del PSI individuò la novità politica nella responsabilità di cui tutti i partiti riuniti insieme si facevano carico. Soltanto i democristiani erano di parere diverso e si affrettarono a ridimensionare la portata politica dell'evento definendo quel Vertice di maggioranza «solo un fatto eccezionale legato a vicende eccezionali»5. L'eccezione, in realtà, sarebbe poi diventata una regola in tutta la fase della solidarietà nazionale, giacché il PCI, oltre a farsi direttamente promotore di due tra le più complesse e travagliate verifiche di governo, prese parte anche ai diversi summit di maggioranza che si susseguirono in quegli anni.

E passiamo così a quelli che sono i partecipanti ai Vertici, distinguendo attori che rivestono ruoli solo partitici e attori anche governativi. Un primo dato riguarda l'elevato numero di incontri, ben il $73 \%$, a cui hanno preso parte, oltre al presidente del Consiglio, politici che rivestivano soltanto incarichi di partito.

Ovviamente, per quanto concerne i Vertici di crisi, trattandosi di una condizione in cui non vi è ancora un governo, gli attori non possono che essere solo partitici. Essi, infatti, sono tenuti dal nuovo presidente del Consiglio (il presidente incaricato) e dai rappresentanti dei partiti che intendono partecipare alla nuova maggioranza di governo.

5 «Corriere della Sera», 18 dicembre 1976. 
TAB. 1. Tipi di Vertice per tipi di attore

\begin{tabular}{lcccc}
\hline Tipi di Vertice & Tipi di attori & Solo partitici & Anche governativi & Tot. \\
\hline Monotematico & & & \\
Pluritematico & 12 & 18 & 30 \\
Dicrisi & 31 & 5 & 36 \\
Tot. & 19 & 0 & 19 \\
\hline
\end{tabular}

Più interessante è invece rilevare che, mentre i Vertici pluritematici nell' $86 \%$ dei casi vedono il capo dell'esecutivo accompagnato solo da uomini di partito, al $60 \%$ dei Vertici monotematici prendono parte anche componenti del governo.

Se, dunque, ben più della metà dei Vertici monotematici vedono la presenza di componenti dell'esecutivo, si tratta per lo più dei titolari dei ministeri interessati alla tematica in discussione - spesso dei ministeri economici. Come era prevedibile, cioè, questo tipo di Vertice è quello che lascia più spazio alle competenze ministeriali.

Il dato più interessante a proposito dei partecipanti riguarda però il mutamento avvenuto a cavallo tra i due decenni considerati: mentre fino alla fine degli anni '70 i partecipanti sono più o meno equamente distribuiti tra attori solo partitici e attori anche governativi, a partire dal 1978 sembrano prevalere i Vertici solo partitici. Più precisamente, dal 1978 tutti i Vertici pluritematici e la maggior parte di quelli monotematici diventano riunioni «ristrette» tra il presidente del Consiglio e i segretari dei partiti della maggioranza.

Si tratta, a primo avviso, di un andamento tutt'altro che sorprendente dal momento che sembrerebbe confermare la crescente - e da più parti deplorata - partitizzazione della politica italiana. Ancora una volta, dunque, il processo decisionale dell'esecutivo italiano risulterebbe dipendere quasi esclusivamente dalla capacità dei partiti della coalizione di trovare un accordo in questi incontri extra-costituzionali. $\mathrm{Ma}$, forse, vale la pena qui di cercare una spiegazione meno semplicistica. Questo mutamento, infatti, va innanzitutto inserito nel più generale trend di verticizzazione e personalizzazione della politica e ci fa porre l'attenzione su una tendenza dei Vertici di maggioranza verso una configurazione che, in realtà, potremmo definire governativa più che partitica. 


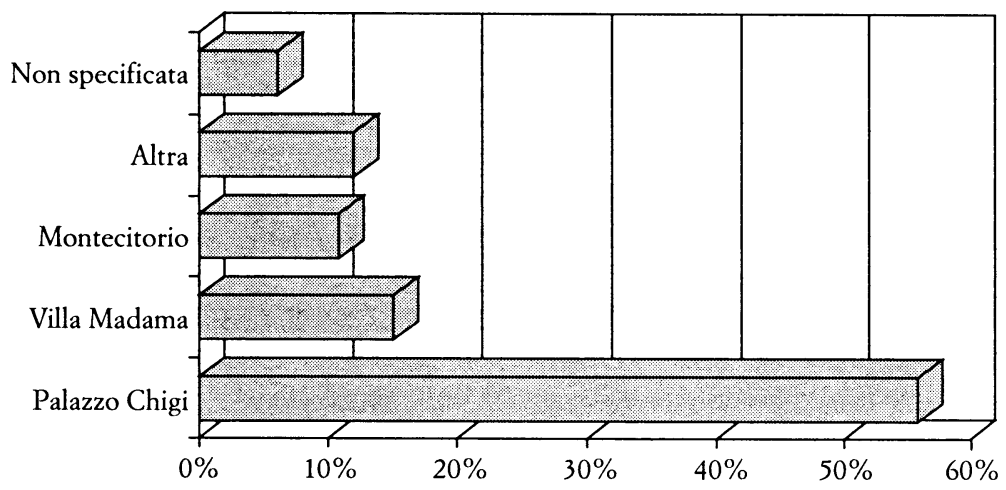

FIG. 2. Distribuzione dei Vertici di maggioranza per sede.

I Vertici diventano infatti riunioni ristrette in quanto non compaiono più, ad esempio, quelle delegazioni di partito così numerose che negli anni ' 70 facevano salire fino a 40 il numero dei partecipanti: i veri protagonisti dell'ultimo decennio sono il presidente del Consiglio e i segretari dei partiti che compongono la maggioranza di governo. La figura stessa di segretario di partito - come abbiamo visto - sembra assumere un ruolo diverso proprio negli anni ' 80 anche perché viene a coincidere con quella di presidente del Consiglio, in una ripetuta sovrapposizione di leadership governativa e leadership di partito che non si era mai verificata nella storia dei Vertici di maggioranza ma che anzi, in qualche occasione, era stata accuratamente evitata.

Riguardo poi alla sede prescelta, come è possibile vedere dalla figura 2, ben il $56 \%$ dei Vertici si sono svolti a Palazzo Chigi, sede ufficiale della presidenza del Consiglio. Se poi aggiungiamo a questa percentuale quella dei Vertici tenutisi a Villa Madama, altra sede di rappresentanza dell'esecutivo, arriviamo ad oltre il $70 \%$.

La maggior parte di questi incontri, dunque, si è svolta nei due luoghi più importanti e rappresentativi del governo: il Vertice di maggioranza come strumento decisionale sembra così sostituire il Consiglio dei Ministri proprio a partire dalla sede.

Per quanto concerne invece la durata dei Vertici va detto che nella gran parte dei casi essi si risolvono in un'unica seduta. Ciò lascia supporre, data la complessità dei temi trattati, una considerevole capacità dei politici - tanto di partito che di go- 
verno - di preparare il terreno in sedi più informali, capacità accompagnata dalla esigenza di presentare il risultato finale delle contrattazioni in un solo incontro definitivo. In parecchi casi il risultato viene infatti raggiunto grazie a diversi contatti, spesso definiti «pre-vertici», che hanno carattere istruttorio. Le cosiddette verifiche sono, senza dubbio, l'esempio più illuminante: questo tipo di vertice, infatti, costituisce generalmente la sede finale in cui accordi discussi in precedenza durante incontri personali, contatti telefonici, scambi di lettere e riunioni bilaterali tra i segretari dei partiti, vengono definitivamente decisi.

A questo discorso è, infine, legato quello concernente l'ultima variabile che si è considerata in questa ricerca, l'esito finale. Anche in questo caso molto dipende dagli accordi e dalle contrattazioni informali che spesso precedono un Vertice. Più dell' $80 \%$ dei summit ha un esito positivo, si conclude, cioè, con un accordo della maggioranza. Il Vertice sembra davvero la «bacchetta magica» che consente ai componenti della coalizione di trovare l'intesa ${ }^{6}$. Se, quindi, consideriamo i Vertici di maggioranza anche dal punto di vista dell'esito, abbiamo un buon indicatore della capacità dei partiti di gestire l'accordo di coalizione e, quindi, indirettamente, della discreta o cattiva salute dell'esecutivo.

Molto spesso agli incontri ha fatto seguito la diramazione agli organi di informazione di una sorta di bollettino finale - a volte un vero e proprio documento ufficiale - in cui venivano delineati i termini della cooperazione. $\mathrm{E}$ non di rado puntuali rapporti dei Vertici sono stati pubblicati anche nella rivista ufficiale della Presidenza del Consiglio dei Ministri. È molto facile, inoltre, che la stampa riportasse anche le dichiarazioni dei segretari politici rilasciate alla fine dei summit e che queste tendessero ad enfatizzare la portata degli accordi. Ad esempio, nel caso del Vertice pluritematico del 5 agosto 1991, svoltosi durante l'ultimo governo Andreotti, in un articolo che ne riportava la cronaca e il cui titolo recitava semplicemente «Raggiunta l'intesa politica di massima», così i diversi protagonisti ne giudicavano l'esito: «riunione positiva», «rinnovato impegno», «consolidamento complessivo della maggioranza», «concorde intesa», «rafforzato il governo a quattro», «spirito di solidarietà della coalizione», «segnale di rafforzamento», «notevoli risultati». Eravamo alla vigilia dell'esplosione di Tangentopoli.

6 Luigi La Spina, «Corriere della Sera», 26 febbraio 1981. 


\section{La fase di «transizione istituzionale»}

Durante l'arco di tempo considerato, il processo decisionale del governo italiano è stato prevalentemente il risultato di informali negoziazioni partitiche piuttosto che di autonome e pianificate decisioni del Consiglio dei Ministri.

Il fatto che i partiti politici possono «dall'esterno» ridurre il campo dei problemi gestiti dai membri del governo non è certo tipico solo del caso italiano. Ovviamente la differenza tra ciascun paese deve essere individuata in quella che è la quantità di influenza esercitata dai partiti, nonché nei mezzi e nei modi in cui le relazioni tra partiti e vertici dell'esecutivo sono organizzati ed espressi ${ }^{7}$. In Italia, il metodo del coordinamento intragovernativo e della collegialità è stato sostituito da una contrattazione quași esclusivamente partitica. E i Vertici di maggioranza sono stati l'espressione di questo metodo. Nel tempo, queste arene informali sono diventate via via più istituzionalizzate così come ha dimostrato l'analisi del loro numero, composizione, contenuto, sede ed esito. Senza però sfüggire alla crisi più generale che ha investito il controllo dei partiti sul processo decisionale.

Il declino dei Vertici era cominciato nei primi anni '80, quando cioè più forte era diventata la polemica contro la partitocrazia, ossia contro l'occupazione partitica delle istituzioni e dei centri di potere. I Vertici tra i partiti vennero fatti bersaglio dai mass media all'interno di un vivace dibattito che si svolse sulle pagine dei maggiori quotidiani nazionali e che non a caso prese spunto dall'idea attribuita a Bruno Visentini della formazione di un governo di tecnici sganciato dal potere invadente dei partiti. I Vertici di maggioranza sembravano sopravvivere quasi come l'ultima roccaforte rimasta ai partiti per contare all'interno di un esecutivo che si andava invece caratterizzando per una sempre più accentuata ed autonoma monocraticità $(\mathrm{Ca}$ lise 1994).

Non sorprende allora che fossero appunto i due primi governi «tecnici», presieduti rispettivamente da Giuliano Amato e Carlo Azeglio Ciampi, a portare a una drastica inversione di rotta.

Con il governo Amato, infatti, i partiti sembrarono aver de-

7 Jean Blondel parla a questo proposito di gabinetti «autonomi» e gabinetti «subordinati» a seconda della portata del controllo partitico; cfr. Blondel $(1989,217-220)$. 
finitivamente perso molte delle prerogative che avevano fatte proprie negli anni passati. Amato, come presidente del Consiglio, ha frequentemente fatto uso del voto di fiducia per verificare in Parlamento l'accordo della maggioranza di governo; si è inoltre assunto la piena responsabilità, in prima persona, delle scelte fatte in merito al rimpasto di ben sette ministri e ha sostituito con periodici incontri con il capo dello stato le riunioni bilaterali con i segretari dei partiti prima così frequenti. Infine, ha preso numerose ed importanti decisioni di politica economica senza previe contrattazioni con i partiti, anzi, pubblicizzandone spesso l'autonomia di decisione: «durante il mio governo non ci furono incontri con gli esperti dei partiti, né sugli indirizzi generali, né sui singoli disegni di legge. Soltanto consultazioni con i gruppi parlamentari sia su materie di indirizzo, sia sul merito di singoli disegni di legge del governo, allo scopo di facilitarne l'approvazione da parte delle camere» (Amato 1994, 366). La stessa crisi di governo seguita alle sue dimissioni nella primavera del 1993 non è stata gestita da un Vertice di maggioranza e l'esecutivo che gli è succeduto, presieduto da Carlo Azeglio Ciampi, si è presentato, sin dall'inizio, come governo non vincolato alle segreterie di partito. Obiettivi importanti come la prosecuzione di riforme in tema di privatizzazioni o come il riassetto di vari settori della pubblica amministrazione, sono stati raggiunti senza ricorrere ad alcun tipo di Vertice. Ciampi, infatti, non ha mai convocato un Vertice di maggioranza con le caratteristiche di quelli rilevati nel ventennio che lo ha preceduto, ma ha soltanto condotto delle consultazioni informali a Palazzo Chigi con i capigruppo dei partiti, attraverso il ben noto sistema degli incontri bilaterali che si sono intensificati prima della presentazione alle Camere di una contestatissima legge finanziaria ${ }^{8}$. Legge finanziaria di cui, come lo stesso Ciampi aveva avuto premura di annunciare alla stampa, i partiti avevano avuto notizia direttamente dai giornali ${ }^{9}$. Titoli dei quotidiani come «Scelgo i ministri senza consultare i partiti» o «Ho messo in riga i partiti» hanno fatto da cornice al quadro appena delineato. La parola «Vertice», usata comunque spesso dai mass media durante il suo governo, si riferisce invece ai numerosi incontri interministeriali che questo premier ha condotto sui temi

8 Sul governo Ciampi cfr. Chimenti (1994) e Pasquino e Vassallo (1994).

9 Cfr. Finanziaria senza partiti, in «Corriere della Sera», 12 settembre 1993. 
più svariati, dal ruolo dei servizi segreti, al mercato del lavoro, alla manovra «antideficit», ai problemi di protezione civile. Ciampi, cioè, nel ridurre al minimo i contatti con i partiti, incontrandosi eventualmente soltanto con i capigruppo parlamentari, ne ha per così dire rivalutato la dimensione rappresentativa, ma solo quella. Riportando invece, nell'ambito dell'esecutivo, con il reiterato ricorso a vertici interministeriali, la soluzione dell'eterno problema della collegialità.

\section{I primi Vertici della Seconda Repubblica}

Eppure, il sipario che sui Vertici di maggioranza sembrava essersi chiuso per sempre si è riaperto alle prime luci dell'alba della cosiddetta Seconda Repubblica, smentendo ogni previsione rivoluzionaria e dando ragione a chi aveva parlato, più saggiamente, di «assetto di crisi, legato a condizioni del tutto eccezionali e transitorie» (Cotta 1994, 135).

Le elezioni del marzo '94 hanno visto la vittoria di una coalizione di centro-destra, formata da alcune forze politiche - la Lega e Forza Italia - che non vogliono assolutamente essere definite partiti, avendo fatto della loro campagna elettorale una vigorosa battaglia contro la partitocrazia. Ciononostante, queste forze, a partire dalla nomina dei ministri del nuovo esecutivo guidato da Silvio Berlusconi, hanno gestito il loro potere di coalizione con uno strumento politico niente affatto nuovo o rivoluzionario: il Vertice di maggioranza.

Questi incontri tra il presidente del Consiglio e gli altri leader delle formazioni politiche della maggioranza hanno avuto, dai mezzi di informazione, l'attenzione di sempre. Ne sono stati registrati i contenuti, la sede, la durata, l'esito con una dovizia di particolari pari a quella dei Vertici che per il ventennio precedente hanno profondamente segnato il funzionamento del nostro sistema politico.

In particolare, nei sette mesi del governo Berlusconi, il termine «Vertice di maggioranza» - così come «verifica» - è ricomparso con frequenza nella titolazione della stampa quotidiana. E stato così possibile registrare, consultando la stessa fonte del ventennio precedente, e cioè il «Corriere della Sera», svariati incontri tra Berlusconi e rappresentanti di partito e/o di governo ai quali è stato dato questo nome. In realtà, se scartiamo - come peraltro abbiamo fatto nella prima parte della ricerca 
per il periodo 1970-1992 - le riunioni informali cosiddette bilaterali tra il presidente del Consiglio e i segretari della maggioranza, sempre numerose in tutti i governi di coalizione, i Vertici veri e propri sono sette.

Anche quelli che non annoveriamo tra i Vertici di maggioranza, tuttavia, presentano delle caratteristiche interessanti. Il caso senz'altro più eclatante è stato l'incontro - che ha rischiato addirittura di far cadere il governo - svoltosi in casa di Berlusconi, tra il ministro della Difesa Previti, il sottosegretario alla Presidenza del Consiglio Letta e alcuni legali di dirigenti della Fininvest. La notizia creò molto scalpore e il dubbio che si trattasse di un vertice di governo o invece di un incontro privato, come in tutta fretta si premurarono di precisare i partecipanti, non impedì che il ministro dei Rapporti con il Parlamento Giuliano Ferrara minacciasse le dimissioni se non fosse stato fornito un chiarimento ufficiale dell'accaduto.

In più di un'occasione, poi, Berlusconi si è incontrato con uno degli alleati di governo, Umberto Bossi, ed in tutti questi casi gli incontri sono stati definiti Vertici ed hanno avuto molto risalto sui mass media ${ }^{10}$. Un altro degli incontri «bilaterali» di Berlusconi che il «Corriere della Sera» registra con evidenza è quello con il segretario dei popolari Buttiglione, durante il quale si discusse di eventuali e possibili alleanze con la maggioranza di governo. In questo caso la sede prescelta è stata la sede romana centrale di Forza Italia. E anche qui, è stato usato il termine vertice.

Oltre a questi incontri assolutamente informali, con il primo governo della Seconda Repubblica si è avuto, come dicevamo, un numero comunque considerevole di Vertici veri e propri, soprattutto tenuto conto della durata dell'esecutivo: ben sette Vertici in soli sette mesi.

Vediamo ora quali sono le loro caratteristiche. Il primo dato interessante è che si tratta di un esecutivo i cui ministri vengono nuovamente designati in sede di Vertice di maggioranza (in

10 Addirittura uno di questi, quello che si svolse nella notte tra il 13 e il 14 agosto 1994 nella villa di Arcore di Berlusconi, ebbe una sorta di appendice in giardino, dove $\mathrm{i}$ due leader si soffermarono a lungo a passeggiare in apparente piacevole compagnia, il tutto naturalmente ampiamente ripreso dalle telecamere televisive. Stesso risalto ebbe anche il secondo di questi tentativi di riappacificazione tra i due leader della coalizione, avvenuto questa volta in Sardegna, in pieno clima vacanziero, nella villa di Berlusconi. Villa che, prima di Bossi, aveva ospitato i ministri Maroni e Previti. 
due diverse sedute), del tipo che abbiamo definito «di crisi», come la maggior parte di quelli del ventennio analizzato in precedenza. Dei restanti Vertici, quattro sono stati monotematici e uno pluritematico. I monotematici hanno affrontato, nell'ordine, la programmazione economica, il tema delle pensioni, il problema del rapporto tra potere esecutivo e magistratura, e poi di nuovo le pensioni all'interno della legge finanziaria. Cinque Vertici si sono svolti a Palazzo Chigi, e due nella residenza privata del presidente del Consiglio, in via dell'Anima a Roma. Il proponente di questi Vertici è stato quasi sempre Berlusconi, tranne nel caso del Vertice pluritematico, proposto da Gianfranco Fini, coordinatore di Alleanza Nazionale. I partecipanti sono solo partitici in due Vertici, quello svoltosi per decidere la compagine ministeriale e quello sulla programmazione economica. In entrambi erano presenti soltanto Berlusconi, Bossi e Fini. Agli altri Vertici hanno invece partecipato attori anche governativi, innanzitutto il vicepresidente del Consiglio e il sottosegretario alla Presidenza e poi, a seconda delle tematiche affrontate, anche i ministri economici e dei ministeri più «caldi» quali Giustizia ed Interni. Infine, a proposito dell'esito di questi incontri, bisogna registrare che tutti si sono conclusi con un accordo della maggioranza.

In cosa dunque sono cambiati i Vertici di maggioranza della Seconda Repubblica - se sono cambiati - rispetto al ventennio precedente?

Andiamo per ordine. Innazitutto la frequenza. Durante il governo Berlusconi, infatti, si registra una media di un Vertice formale al mese, senza contare, come abbiamo visto, gli incontri informali, i summit a due o a tre, le cene private, ecc. Proprio il livello di formalizzazione, e quindi gli indicatori del processo di istituzionalizzazione che avevamo rilevato nel ventennio precedente vengono a mancare, e questo vale per quasi tutte le variabili che abbiamo analizzato, come vedremo tra poco.

Il secondo dato interessante riguarda la tipologia: se la ricomparsa del Vertice di crisi per la formazione di un governo di coalizione con partner piuttosto rissosi appare scontata, meno ovvia è la distribuzione dei Vertici a seconda che si sia trattato di incontri pluri o monotematici. Tutti i Vertici tranne uno sono infatti stati monotematici e se a ciò si aggiunge il fatto che l'unico pluritematico era nato in realtà per discutere soltanto la questione delle nomine RAI, si vede come i Vertici veri e propri del governo Berlusconi siano stati dedicati solo ad issues 
specifiche, prevalentemente di politica economica. Le cosiddette verifiche degli accordi di maggioranza e comunque tutti gli incontri dedicati più in generale alla politica coalizionale sono stati invece discussi in quelle sedi più informali cui abbiamo fatto riferimento prima.

Un altro cambiamento di rilievo concerne poi la sede prescelta. Sempre più spesso, come abbiamo visto, questa si è spostata da Palazzo Chigi - o da altre sedi dell'esecutivo - alla dimora privata del presidente del Consiglio. La sua villa di Arcore così come la sua casa romana in via dell'Anima si incontrano parecchie volte nei resoconti della stampa, essendo infatti le sedi prescelte per la maggior parte degli incontri informali tra $\mathrm{i}$ leader della maggioranza anche nel caso delle forze politiche che appoggiano il governo dall'esterno, come ad esempio i Riformatori di Marco Pannella; insomma, di tutti coloro che nell'arco dei sette mesi del governo Berlusconi hanno discusso con il capo di Forza Italia delle possibili alleanze anche in vista di una crisi di governo sempre annunciata e poi verificatasi nel dicembre del ' 94.

Mai la personalizzazione della politica aveva avuto fino a questo momento caratteristiche simili e così pubblicizzate dai mass media. Sicuramente è la prima volta nella storia d'Italia che l'indirizzo della residenza privata del presidente del Consiglio diventa nell'immaginario dell'opinione pubblica il luogo dove si decidono le sorti del paese. Stampa e televisione non mancano infatti di riportare i resoconti di queste riunioni, con tutte le notizie di contorno: ad esempio della maggior parte degli incontri informali nelle varie case del presidente del Consiglio vengono spesso sottolineati gli aspetti per così dire conviviali, arrivando persino a riportare i menù dei pranzi e delle cene con una sorprendente dovizia di particolari. Lo stesso «Vita Italiana» registra gli incontri tenutisi a casa di Berlusconi come Vertici di maggioranza, al pari di quelli svolti a Palazzo Chigi.

Per ciò che invece concerne i partecipanti a questi Vertici di maggioranza, notiamo che, se da un lato i numerosi incontri a cui prendono parte solo i leader dei partiti che compongono la coalizione di governo farebbero pensare ad una continuità rispetto a quella collegialità ristretta che abbiamo rilevato negli anni '80, non bisogna invece trascurare il fatto che la maggior parte dei Vertici del governo Berlusconi, che vedono la partecipazione tanto di attori con incarichi «solo partitici» quanto di 
attori «anche governativi», riunisce un considerevole numero di persone. Accanto al presidente del Consiglio, sempre presente, vi è il sottosegretario alla Presidenza e, altra presenza costante, il vicepresidente del Consiglio. Questa figura, in particolare, è tornata in auge dopo i governi Amato e Ciampi nel suo ruolo di bilanciamento all'interno della coalizione. Non mancano mai i segretari dei partiti che formano la coalizione, in un caso vi sono i capigruppo al Parlamento e qualche volta vi ha preso parte anche Marco Pannella, in qualità di rappresentante del gruppo dei Riformatori che appoggiava la coalizione dall'esterno. Molte volte poi compaiono i ministri dei dicasteri più importanti come quelli economici. Insomma, ci troviamo di fronte nuovamente a riunioni numerose che testimoniano l'alto livello di frammentazione della politica governativa, con una collegialità ministeriale praticamente inesistente. Ancora, la frammentazione viene accentuata da un fatto assolutamente nuovo nella storia dei Vertici: la partecipazione discontinua di uno dei leader della maggioranza, Umberto Bossi. Il segretario della Lega, uno dei responsabili principali dei numerosi dissidi all'interno della maggioranza e poi, di fatto, artefice della crisi di governo, non ha preso parte a tutti i Vertici, dando sempre molto risalto, con l'aiuto dei media, alla sua non partecipazione.

\section{Un tentativo di interpretazione}

I Vertici di maggioranza che abbiamo rilevato e analizzato in questi venticinque anni non sostituiscono certo il governo nel momento decisionale. Non si può dire, cioè, che il Vertice abbia fatto le veci dei numerosi esecutivi che si sono succeduti dal 1970 ad oggi. Una cosa è però certa: l'incontro tra governo e partiti formalizzato nei tempi, nei modi e nelle procedure che abbiamo visto, non può essere considerato semplicemente e semplicisticamente uno strumento normale di politica coalizionale. Esso, in molte occasioni, ha infatti parzialmente agito da surrogato dell'azione di governo vera e propria. La maggior parte dei Vertici di maggioranza ha preceduto un Consiglio dei Ministri e questo non è successo solo nel caso dei Vertici in cui si discuteva di politica coalizionale, per cui è intuitivo pensare che i partner della maggioranza che formano il governo vogliano verificare e consolidare la loro intesa prima di un Consiglio decisivo. Il punto è invece che molte decisioni prese in sede di 
Consiglio dei Ministri spesso hanno ratificato decisioni prese in sede di Vertice, magari in un Vertice monotematico convocato ad boc.

In un governo rissoso come può esserlo un governo di coalizione, $i$ veri momenti collegiali sono garantiti da questi incontri informali ma non troppo (non dimentichiamo l'ufficialità implicita ad esempio nella presenza costante del presidente del Consiglio e nella scelta della sede).

Il trend temporale interessante, da questo punto di vista, è il passaggio da una collegialità molto ampia, che vedeva riuniti rappresentanti dell'esecutivo e rappresentanti dei partiti fino agli inizi degli anni ' 80 , ad una sorta di «collegialità ristretta», che vede riuniti nel decennio successivo soprattutto il presidente del Consiglio e i soli segretari dei partiti che formano la coalizione di governo. Questo è spiegabile con due ordini di motivi. Il primo è quello che riguarda il ruolo rappresentativo dei partiti che a mano a mano viene a mancare di spessore e di importanza. Tanto la figura del capogruppo al parlamento quanto quella di vicesegretario del partito comparivano infatti con regolarità nei Vertici del centro-sinistra così come in quelli della cosiddetta fase della solidarietà nazionale. Il secondo ordine di motivi riguarda invece la tendenza - non solo italiana - ad una forte verticizzazione e personalizzazione della politica. Si tratta di una tendenza dettata anche da esigenze di politica internazionale: gli incontri tra i paesi industrializzati, il nuovo rapporto tra est e ovest del mondo, le nuove relazioni tra i paesi dell'Unione Europea sono tutte occasioni nelle quali ciascun paese ha l'esigenza di presentarsi con un esecutivo certo non frammentato e litigioso. E, soprattutto, con un capo del governo dotato di un certo carisma o almeno di una certa credibilità. La crisi dell'organismo collegiale Consiglio dei Ministri, il ricorso sempre più frequente ad organi ristretti e la tendenza a quella che è stata definita la «presidenzializzazione» dell'esecutivo (Cassese 1993), sono infatti fenomeni che si riscontrano in quasi tutti i moderni sistemi parlamentari di governo. E che contribuiscono a spiegare quello che è il secondo elemento di trasformazione che emerge dall'analisi dei Vertici di maggioranza degli ultimi venticinque anni: il diverso ruolo che in essi riveste il presidente del Consiglio. Sembra chiaro infatti che in questo trend di verticizzazione della politica nel quale anche questi summit si trasformano in una sorta di informali inner cabinets, il ruolo che esce rafforzato è proprio quello del capo dell'esecu- 
tivo. $\grave{E}$ indubitabile insomma che tale collegialità ristretta non avvantaggi certo i partiti la cui crisi, come sappiamo, negli anni ' 80 ha esiti sempre più vistosi. Gli stessi segretari di partito, sempre presenti in ogni Vertice, rivestono un ruolo che non può non apparire secondario rispetto a quello di un primo ministro che sembra aver guadagnato un più ampio margine di manovra rispetto agli anni passati.

O meglio, è sicuramente il presidente del Consiglio il primo a trarre vantaggio dal vuoto di potere che lasciano i partiti della Prima Repubblica come appare chiaro dalla conduzione degli esecutivi di Amato e Ciampi. I due governi della cosiddetta fase di transizione istituzionale, come abbiamo visto, sono nati entrambi sotto il segno della separatezza dai partiti ed entrambi i presidenti del Consiglio hanno visto valorizzati i loro poteri di direzione della politica generale dell'esecutivo. Si tratta tuttavia di innovazioni tutte interne ad una situazione politica generale quanto meno anomala. Come è stato giustamente osservato, infatti, «il Governo autonomo dai partiti è, in presenza di una crisi gravissima della partitocrazia, un Governo capace di realizzare politiche pubbliche innovative, ma resta pur sempre un Governo politicamente debole perché privo di una sicura fonte di legittimazione politica» (Pitruzzella 1994, 231).

Meno potere ai partiti e più potere al primo ministro non è dunque la ricetta per risolvere il problema della collegialità dell'esecutivo, e più in generale della governabilità. Anzi, la caduta del governo Berlusconi, secondo Andrea Manzella, «è stata l'ultima prova dell'impossibilità di superare la tradizionale debolezza dei nostri governi di coalizione, attraverso la mera auctoritas politica del presidente del Consiglio. E quindi la definitiva conferma che la stabilità dei governi può fondarsi solo su una plusvalenza istituzionale del premier, cioè sulla sua legittimazione diversa - seppure non contraddittoria - e rispetto al Parlamento e rispetto ai suoi ministri» (Barbera et al. 1995, 238).

Né, d'altronde, la nuova legge elettorale parrebbe aver fornito al capo dell'esecutivo questa legittimazione. Gran parte delle aspettative suscitate dalla riforma maggioritaria sono state infatti deluse e la nuova classe politica nata dalle elezioni del marzo '94 non sembra aver comportato «né una chiara identificazione dei «responsabili» di governo, essendo perdurate le coalizioni di governo, né un incremento della stabilità governativa, né soprattutto l'avvio di una politica innovativa rispetto al passato» (Ignazi e Katz 1995, 35). 
Oggi, infatti, con il nostro sistema elettorale i governi continuano ad essere governi di coalizione e la tendenza verso una presidenzializzazione del governo non ha evitato - come abbiamo visto - che la pratica dei Vertici di maggioranza si riproponesse ancora una volta. La stessa fase di formazione del primo governo nato dopo il voto del 27 e 28 marzo è infatti stata gestita con una politica di coalizione spartitoria molto simile ai governi che hanno preceduto la XII Legislatura (Ceccanti e Fabbrini 1995) con una compagine ministeriale organizzata sostanzialmente in un Vertice di maggioranza. E, se i numerosi contrasti tra i ministri sono stati formalmente arginati dalla nomina da parte del presidente del Consiglio di un «portavoce ufficiale del governo», è in realtà con il ricorso ai Vertici che in pratica si è cercato di far fronte ad un livello di collegialità tra i più bassi che siano mai stati registrati. Ma con un cambiamento di rilievo rispetto al passato: i tentativi di ricomporre l'alto livello di rissosità all'interno della compagine governativa sono stati sempre fatti in incontri assolutamente informali tra il presidente del Consiglio e i vari partner della coalizione, con un lavoro di mediazione di cui Berlusconi è apparso essere il principale artefice. Come egli stesso ha dichiarato alla stampa: «su tutti gli argomenti capaci di suscitare una crisi faccio io, prima delle riunioni, un lavoro di tessitura» ${ }^{11}$.

I Vertici di maggioranza sono stati invece dedicati a temi specifici del programma di governo e soprattutto a quelli, particolarmente scottanti, di politica economica. Come se, insomma, i Vertici della Seconda Repubblica si fossero caratterizzati come arena politica governativa più che coalizionale, anche se con il livello di frammentazione di cui abbiamo sottolineato la consistenza.

E infatti, né questi mutamenti nei Vertici di maggioranza, né, però, quelli più generali e di più grossa portata quali il ridimensionamento del ruolo dei partiti, una tendenza alla presidenzializzazione dell'esecutivo e il nuovo sistema elettorale tutti passaggi presentati spesso come indispensabili per la soluzione degli annosi problemi riguardanti la governabilità del nostro paese - hanno per ora portato ad un diverso funzionamento della politica governativa rispetto al passato. Con una continuità testimoniata, tra le altre cose, proprio dai Vertici di mag- 
gioranza. Tornati ad essere protagonisti della Seconda Repubblica come lo furono della Prima.

\section{Riferimenti bibliografici}

Amato, G. (1994), in Un governo nella transizione. La mia esperienza di Presidente del Consiglio, in «Quaderni Costituzionali», n. 3, pp. 355-371.

Andreotti, G. (1991), Governare con la crisi, Milano, Rizzoli.

Barbera, A., A. Manzella, S. Bartole e F. Lanchester (1995), La forma di governo in transizione: interventi, in «Quaderni Costituzionali», n. 2, pp. 211-256.

Blondel, J. (1989), Decisioni di governo e vincoli partitici, in «Rivista Italiana di Scienza Politica», XIX, n. 2, pp. 199-222.

Blondel, J. e F. Müller-Rommel (a cura di) (1988), Cabinets in Western Europe, London, Macmillan.

Bogdanor, V. (a cura di) (1983), Coalition Government in Western Europe, London, Heinemann.

Budge, I. e H. Keman (1990), Parties and Democracy, Oxford, Oxford University Press.

Caciagli, M., F. Cazzola, L. Morlino e S. Passigli (a cura di) (1994), L'Italia tra crisi e transizione, Roma-Bari, Laterza.

Calandra, P. (1986), Il Governo della Repubblica, Bologna, Il Mulino.

Calise, M. (1994), Il governo tra istituzione e politica, in Caciagli, Cazzola, Morlino e Passigli (1994), pp. 141-162.

Capotosti, P.A. (1975), Accordi di governo e Presidente del Consiglio dei Ministri, Milano, Giuffrè.

Cassese, S. (1993), Il potere esecutivo nei sistemi parlamentari di governo, in «Quaderni Costituzionali», n. 1, pp. 141-148.

- (1995), Maggioranza e minoranza. Il problema della democrazia in Italia, Milano, Garzanti.

Ceccanti, S. e S. Fabbrini (1995), Transizione verso Westminster? Ambiguità e discontinuità nella formazione del Governo Berlusconi, in G. Pasquino (a cura di), L'alternanza inattesa. Le elezioni del 27 marzo 1994 e le loro conseguenze, Soveria Mannelli, Rubbettino, pp. 257-283.

Chimenti, C. (1994), Il Governo dei professori, Firenze, Passigli.

Cotta, M. (1988), Italy: A Fragmented Government in Blondel e Müller-Rommel (1988), pp. 120-137.

- (1994) Il governo di partito in Italia. Crisi e trasformazione dell'assetto tradizionale, in Caciagli, Cazzola, Morlino e Passigli (1994), pp. 119-139.

Criscitiello, A. (1993), Majority Summits: Decision-making inside the Cabinet and out. Italy, 1970-1990; in «West European Politics», vol. 16, n. 4, pp. 581-594. 
Cuccodoro, E. (1983), Collegialità ministeriale, comitati e Vertici, in «Rivista Trimestrale di Diritto Pubblico», n. 3, pp. 825-839.

Dunleavy, P. e R.A.W. Rhodes (1990), Core Executive Studies in Britain, «Public Administration», vol. 68, pp. 3-28.

Ferrara, G. (1973), Il governo di coalizione, Milano, Giuffrè.

Hine, D. (1993), Governing Italy. The Politics of Bargained Pluralism, Oxford, Oxford University Press.

Hine, D. e R. Finocchi (1991), The Italian Prime Minister, in G.W. Jones (a cura di), West European Prime Ministers, London, Frank Cass.

Ignazi, P. e R.S. Katz (1995), Introduzione. Ascesa e caduta del governo Berlusconi, in P. Ignazi e R.S. Katz (a cura di), Politica in Italia. Edizione '95, Bologna, Il Mulino.

Jones, G.W. (a cura di) (1991), West European Prime Ministers, London, Frank Cass.

Lavagna, C. (1974), Maggioranza al governo e maggioranze parlamenta$r i$, in «Politica del Diritto», n. 6, pp. 673-697.

Laver, M. e N. Schofield (1990), Multiparty Government. The Politics of Coalition in Europe, Oxford, Oxford University Press.

Mény, Y. (1990), Istituzioni e politica. Le democrazie: Stati Uniti, Francia, Gran Bretagna, Italia e Repubblica Federale Tedesca, Rimini, Maggioli.

Müller-Rommel, F. (1988), Federal Republic of Germany in Blondel e Müller-Rommel, (1988), pp. 151-166.

Pappalardo, A. (1978), Partiti e governi di coalizione in Europa, Milano, Franco Angeli.

Pasquino, G. (1994), Le coalizioni di pentapartito (1980-91): quale governo dei partiti? in Caciagli, Cazzola, Morlino, Passigli (1994), pp. 99-118.

Pasquino, G. e C. Mershon (a cura di) (1994), Politica in Italia. Edizione '94, Bologna, Il Mulino.

Pasquino, G. e S. Vassallo (1994), Il governo di Carlo Azeglio Ciampi, in Pasquino e Mershon (a cura di) (1994), pp. 69-89.

Pitruzzella, G. (1994), Art. 92-93, Il Consiglio dei Ministri, in AA.VV., Commentario della Costituzione, Bologna, Zanichelli.

Pridham, G. (a cura di) (1986), Coalitional Behaviour in Theory and Practice: An Inductive Model for Western Europe, Cambridge, Cambridge University Press.

Rebuffa, G. (1995), La Costituzione impossibile. Cultura politica e sistema parlamentare in Italia, Bologna, Il Mulino.

Rolla, G. (1982), Il Consiglio dei Ministri tra modello costituzionale e prassi, in «Quaderni Costituzionali», II, n. 2, pp. 367-398.

Sartori, G. (1994), Comparative Constitutional Engineering. An Inquiry into Structures, Incentives and Outcomes, London Macmillan, trad. it. Ingegneria costituzionale comparata, Bologna, Il Mulino, 1995.

Vassallo, S. (1994), Il governo di partito in Italia, Bologna, Il Mulino. 